\title{
Time Delay Circuits: A Quality Criterion for Delay Variations versus Frequency
}

\author{
Seyed Kasra Garakoui, Eric A.M. Klumperink, Bram Nauta,Frank E. van Vliet \\ University of Twente, CTIT, ICD group, Enschede, The Netherlands
}

\begin{abstract}
This paper shows that the group delay of a delay circuit does not give sufficient information to predict the delay vs. frequency. A new criterion $\left(f_{\varphi=0}\right)$ is proposed that characterizes the delay variations over a specified frequency range. The mathematical derivation of $f_{\varphi=0}$ for a single delay block and a cascade of delay blocks is shown. As examples the criterion is applied to the design of an $\mathrm{RC}$ and $\mathrm{LC}$ delay block. Delay predictions based on $f_{\varphi=0}$ are compared with simulation results, showing reasonable agreement.
\end{abstract}

\section{INTRODUCTION}

Time delay circuits have wide applications in different systems, such as beamforming systems, delay locked loops, filters and equalizers. In some of these applications it is important to maintain constant delay over a significant frequency band, e.g. for wideband beamforming systems ("phased array") [1]. This is because delay variations result in changes of the beam pattern, such as in beam direction and side lobe levels [1]. As practical delay circuits show some delay variation over frequency, characterizing this variation over the relevant band is important.

The quality of time delay circuits is often evaluated in terms of group delay. In this paper we want to show that group delay is useful, but not sufficient, to characterize delay variation vs. frequency. This paper proposes a criterion that, together with group delay, relates the delay variation and frequency bandwidth to each other.

Fig 1 shows the phase vs. frequency characteristic of an ideal phase shifter and an ideal time delay block with time delay $\tau_{\mathrm{d}}$ (in this paper all the frequency and phase axes are linear).

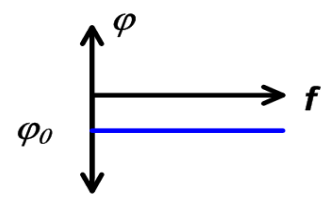

(a)

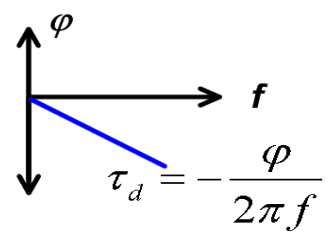

(b)
Figure 1. Phase vs. frequency for (a) an ideal phase shifter and (b) an ideal time delay element

Both an ideal phase shifter and ideal delay block should have unity gain, but they have different group delays versus frequency. Group delay $\left(\tau_{\mathrm{g}}\right)$ is defined as follows [2]:

$$
\tau_{g}=-\frac{1}{2 \pi} \cdot \frac{d \varphi}{d f}
$$

For an ideal phase shifter, the group delay vs. frequency is constant and equal to zero. For an ideal time delay block it is also constant but non-zero, with a group delay equal to the amount of time delay (see Fig. 1).

For a phase shifter block the conditions of zero group delay and unity amplitude gain vs. frequency defines the necessary and sufficient conditions to define the block as an ideal phase shifter. However, the condition of constant group delay and unity amplitude gain vs. frequency does not necessarily correspond to constant time delay. Fig 2 a shows two phase vs. frequency plots which have identical and constant group delay for the frequency range $\left[\mathrm{f}_{0}-\Delta \mathrm{f}, \mathrm{f}_{0}+\Delta \mathrm{f}\right]$. However, only line 1 crosses through the point $(\mathrm{f}=0, \varphi=0)$ which defines the characteristic of an ideal time delay block.
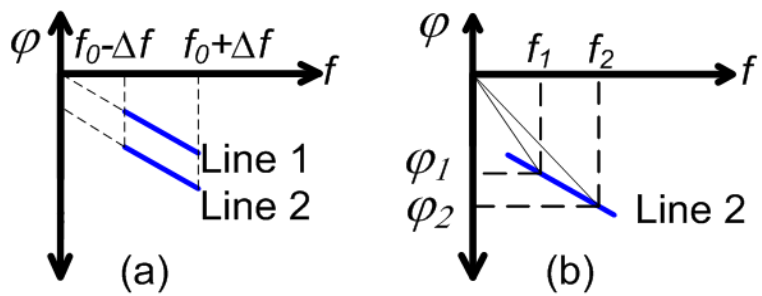

Figure 2. Different phase lines with equal group delay, where only Line 1 defines an ideal time delay b) Different points on Line 2 corresponding to different time delays

For Line 2 the time delay varies with frequency. Fig $2 b$ illustrates this for two example frequencies $f_{1}$ and $f_{2}$, where the time delays are equal to $\tau_{1}=-\varphi_{1} / 2 \pi f_{1}$ and $\tau_{2}=-\varphi_{2} / 2 \pi f_{2}$ respectively. So the group delay is the same, but the time delay is not, which shows that knowledge about group delay is not sufficient to quantify the amount of time delay versus frequency [3],[4].

In section II we propose a new criterion $\mathrm{f}_{\varphi=0}$ that relates the amount of time delay variation to the frequency range. In section III, the amount of time delay variation for circuits with a frequency dependent group delay is analyzed. Section IV gives examples, and derives $\mathrm{f}_{\varphi=0}$ equations for two commonly used time delay circuits: an RC and an LC delay circuit. In section $\mathrm{V}$ we extract $\mathrm{f}_{\varphi=0}$ for a cascade of time delay blocks. In section VI we verify the ability of the proposed criterion $f_{\varphi=0}$ 
to predict the time delay variation for the $\mathrm{RC}$ and $\mathrm{LC}$ delay circuits, while section VII draws conclusions.

\section{A NEW CRITERION $\left(\mathrm{F}_{\varphi=0}\right)$ FOR TIME DELAY CIRCUITS}

Fig 3a gives an example for the phase vs. frequency characteristic of a typical time delay block (e.g. an RC delay circuit). If in the frequency range $\left[\mathrm{f}_{0}-\Delta \mathrm{f}, \mathrm{f}_{0}+\Delta \mathrm{f}\right]$ the transfer function is approximated by a straight line, then two parameters are sufficient to fully characterize this line:

1. The slope of the phase versus frequency, i.e. $-\tau_{\mathrm{g}}$

2. The phase for at least one frequency point

This point can be any point of the line in the relevant frequency band or any point on the linearly extrapolated phase line. We propose to use the point with zero phase where the line crosses the frequency axis and we will call this frequency $\mathrm{f}_{\varphi=0}$. We introduce $\mathrm{f}_{\varphi=0}$ as a new criterion to approximately quantify time delay variations inside the frequency band. The criterion has some interesting characteristics:

1. $\mathrm{f}_{\varphi=0}=0$ corresponds to ideal (constant) time delay behavior inside the band

2. $\mathrm{f}_{\varphi=0}= \pm \infty$ corresponds to ideal (constant) phase shift behavior inside the band.

3. $\mathrm{f}_{\varphi=0} \neq 0$ in combination with constant group delay corresponds to approximate time delay behavior, where the delay variation versus frequency can be predicted accurately.

Fig $3 \mathrm{~b}$ shows two transfer function examples with constant group delay inside the band $\left[\mathrm{f}_{0}-\Delta \mathrm{f}, \mathrm{f}_{0}+\Delta \mathrm{f}\right]$ but with different $\mathrm{f}_{\varphi=0} \neq 0$. We will now relate the time delay variation for these time delay block approximations, based on the center frequency of the band $\left(f_{0}\right)$, the frequency range $( \pm \Delta f)$, the group delay $\left(\tau_{g}\right)$ and the proposed criterion $\left(f_{\varphi=0}\right)$ which is shown in Fig. 4.

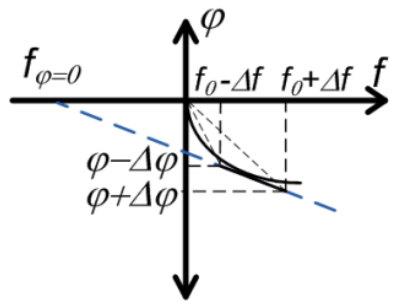

(a)

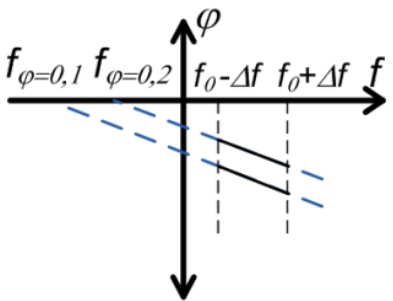

(b)
Figure 3. (a) $\varphi$ vs. $f$ for a frequency range which the curve is modeled as a straight line inside it .(b) $f_{\varphi=0}$ can be used as a criterion to differ $\varphi$ vs. $f$ lines with equal group delays

The amount of time delay at $\mathrm{f}_{0}$ is $-\varphi\left(\mathrm{f}_{0}\right) / 2 \pi \mathrm{f}_{0}$, whereas it is $-\varphi\left(\mathrm{f}_{0}+\Delta \mathrm{f}\right) / 2 \pi\left(\mathrm{f}_{0}+\Delta \mathrm{f}\right)$ at $\mathrm{f}_{0}+\Delta \mathrm{f}$, and $-\varphi\left(\mathrm{f}_{0}-\Delta \mathrm{f}\right) / 2 \pi\left(\mathrm{f}_{0}-\Delta \mathrm{f}\right)$ at $\mathrm{f}_{0}-\Delta \mathrm{f}$. Equation 2 defines the phase versus frequency, depending on group delay $\left(\tau_{\mathrm{g}}\right)$ and $\mathrm{f}_{\varphi=0}$. Equations 3 and 4 show time delays at frequencies $\mathrm{f}_{0}$ and $\mathrm{f}_{0}+\Delta \mathrm{f}$. We can also find the delay at $\mathrm{f}_{0}-\Delta \mathrm{f}$ from equation 2. However, because the $\varphi$ vs. $f$ transfer function of Fig 4 is odd symmetric around $f_{0}$ the absolute delay variation from $\mathrm{f}_{0}-\Delta \mathrm{f}$ to $\mathrm{f}_{0}$ is equal to delay variation from $\mathrm{f}_{0}$ to $\mathrm{f}_{0}+\Delta \mathrm{f}$. Because of this reason, we solve the equations only for upper half part of the frequency.

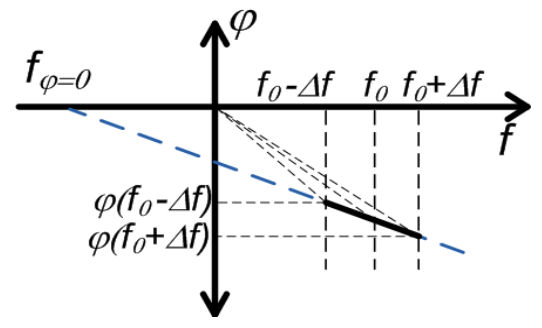

Figure 4. Illustration of a phase characteristic of a delay block which has constant group delay inside $\left[\mathrm{f}_{0}-\Delta \mathrm{f}, \mathrm{f}_{0}+\Delta \mathrm{f}\right]$

$$
\begin{aligned}
& \varphi=-\tau_{g}\left(f_{0}\right) \cdot 2 \pi\left(f-f_{\varphi=0}\right) \\
& \tau_{d}\left(f_{0}\right)=-\frac{\varphi_{0}}{2 \pi f_{0}}=\tau_{g}\left(f_{0}\right)\left(1-\frac{f_{\varphi=0}}{f_{0}}\right) \\
& \tau_{d}\left(f_{0}+\Delta f\right)=-\frac{\left(\varphi_{0}+\Delta \varphi\right)}{2 \pi\left(f_{0}+\Delta f\right)}=\tau_{g}\left(f_{0}\right)\left(1-\frac{f_{\varphi=0}}{f_{0}+\Delta f}\right)
\end{aligned}
$$

The ratio of the delay variation inside the band to the absolute value of the delay at $\mathrm{f}_{0}$ is useful to quantify the relative deviation of the delay that we can get inside the band (sometimes referred to as delay error or delay accuracy). Equation 5 shows this ratio and also an approximation for the condition $\Delta f / f_{0}<<1$. Because group delay is considered constant in the frequency range, Equation 5 is independent of the group delay.

$$
\frac{\tau_{d}\left(f_{0}+\Delta f\right)-\tau_{d}\left(f_{0}\right)}{\tau_{d}\left(f_{0}\right)}=\frac{\frac{f_{\varphi=0}}{f_{0}}-\frac{f_{\varphi=0}}{f_{0}+\Delta f}}{1-\frac{f_{\varphi=0}}{f_{0}}} \approx \frac{\frac{f_{\varphi=0}}{f_{0}}}{1-\frac{f_{\varphi=0}}{f_{0}}} \frac{\Delta f}{f_{0}}
$$

For a block with constant phase in the frequency band, $\mathrm{f}_{\varphi=0}$ becomes infinite and with condition $\Delta \mathrm{f} / \mathrm{f}_{0}<<1$ equation 5 will result in equation 6 :

$$
\left.\frac{\tau_{d}\left(f_{0}+\Delta f\right)-\tau_{d}\left(f_{0}\right)}{\tau_{d}\left(f_{0}\right)}\right|_{\text {Phase_Shifter }} \approx-\frac{\Delta f}{f_{0}}
$$

For an ideal time delay, $\mathrm{f}_{\varphi=0}$ is equal to zero and equation 5 will result in:

$$
\left.\frac{\tau_{d}\left(f_{0}+\Delta f\right)-\tau_{d}\left(f_{0}\right)}{\tau_{d}\left(f_{0}\right)}\right|_{\text {Time_Delay }}=0
$$

In summary, if $f_{\varphi=0}$ is non-zero and the group delay is constant for a circuit, it can be used as an approximation of a time delay circuit. Equation 5 gives the relative variation of the time delay over the band. In the next section we derive the time delay variation for more realistic time delay blocks for which the group delay is frequency dependent. 


\section{TIME DELAY OF BLOCKS WITH FREQUENCY DEPENDENT GROUP DELAY}

Fig 5 shows $\varphi$ vs. f characteristic of a typical practical delay circuit with a group delay that is frequency dependent. If we know the group delay of the characteristic at the center frequency $\mathrm{f}_{0}$ of the band, we can again derive an estimate of the delay for $\mathrm{f}_{0}+\Delta \mathrm{f}$.

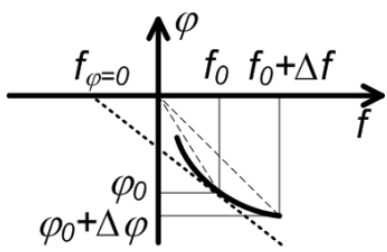

Figure 5. Phase vs. frequency transfer function with a frequency dependent group delay

To this end we draw the slope-line at the point $\left(\mathrm{f}_{0}, \varphi\left(\mathrm{f}_{0}\right)\right)$. This line crosses the $f$-axis at point $f_{\varphi=0}$, and hence the following equation holds:

$$
\left.\frac{\partial \varphi}{\partial f}\right|_{f_{0}}=\frac{\varphi_{0}}{f_{0}-f_{\varphi=0}}
$$

If inside the band, the variation of the phase around $\mathrm{f}_{0}$ is not very large, we can approximate it with the first two terms of a Taylor series. In this case the calculations are identical to those in the previous section and the delay at $\mathrm{f}_{0}+\Delta \mathrm{f}$ can be estimated with equation 4 , while the relative delay variation can be assessed by equation 5 .

If desired, of course a higher order Taylor series also can be used to take into account the effect of the curvature in the phase characteristic on delay as shown by equations 9 and 10:

$$
\begin{aligned}
& \tau_{d}\left(f_{0}+\Delta f\right)=-\frac{\varphi\left(f_{0}+\Delta f\right)}{2 \pi\left(f_{0}+\Delta f\right)} \approx \\
& \approx-\frac{\varphi\left(f_{0}\right)}{2 \pi\left(f_{0}+\Delta f\right)}+\frac{\tau_{g}\left(f_{0}\right)}{\left(f_{0}+\Delta f\right)} \cdot \Delta f+\frac{1}{2} \frac{\left.\frac{\partial \tau_{g}}{\partial f}\right|_{f_{0}}}{\left(f_{0}+\Delta f\right)} \cdot(\Delta f)^{2}
\end{aligned}
$$

If $\Delta \mathrm{f} / \mathrm{f}_{0}<<1$ then this equation can be approximated as:

$$
\tau_{d}\left(f_{0}+\Delta f\right) \approx-\frac{\varphi\left(f_{0}\right)}{2 \pi f_{0}}\left(1-\frac{\Delta f}{f_{0}}\right)+\tau_{g}\left(f_{0}\right) \cdot \frac{\Delta f}{f_{0}}+\left.\frac{1}{2} \cdot f_{0} \cdot \frac{\partial \tau_{g}}{\partial f}\right|_{f_{0}} \cdot\left(\frac{\Delta f}{f_{0}}\right)^{2}
$$

However, for finding time delay, in many cases a linear approximation of the phase vs. frequency curve is enough and there is no need to use the second order term of the Equation 10. In the next section we explain how to find $\mathrm{f}_{\varphi=0}$ at any point of the phase vs. frequency curve.

\section{IV. $\mathrm{F}_{\varphi=0}$ FOR AN RC AND LC CIRCUIT}

There are different types of time delay circuits and based on their structure they show different phase-frequency transfer functions. We will now solve equation 8 for two commonly used examples of delay blocks, an RC delay block and an LCsegment of an infinite LC delay line [5]. If we assume that the operating frequency of an LC delay line is much less than $(2 \pi)^{-1}(\mathrm{LC})^{-0.5}$, the load impedance which every segment sees at its output is real and equal to $\mathrm{Z}_{0}=(\mathrm{L} / \mathrm{C})^{0.5}$, (the "characteristic impedance").

Fig 6 shows an RC delay block and one segment of an LC delay line and their phase vs. frequency transfer functions.

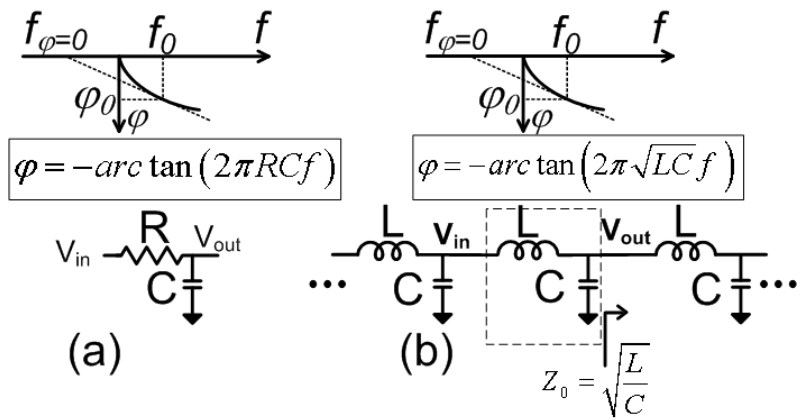

Figure 6. Phase vs. frequency transfer function for (a) an RC delay block and (b) one segment of an infinite LC delay line

By substituting phase vs. frequency characteristics of each block of figure 6 into equation 8 the values of $f_{\varphi=0}$ can be found. This substitution results in equations 11a for an $\mathrm{RC}$ delay block and $11 \mathrm{~b}$ for an LC delay segment.

$$
\begin{aligned}
& \left.\frac{\partial}{\partial f}(-\arctan (2 \pi R C f))\right|_{f_{0}}=-\frac{\arctan \left(2 \pi R C f_{0}\right)}{f_{0}-f_{\varphi=0}} \\
& \left.\frac{\partial}{\partial f}(-\arctan (2 \pi \sqrt{L C} f))\right|_{f_{0}}=-\frac{\arctan \left(2 \pi \sqrt{L C} f_{0}\right)}{f_{0}-f_{\varphi=0}}
\end{aligned}
$$

For an RC delay block the solution of equation 11a in terms of the $\mathrm{f}_{\varphi=0}$ is:

$$
\begin{aligned}
& f_{\varphi_{0}}=f_{0}\left[1-\frac{1+\left(2 \pi R C f_{0}\right)^{2}}{2 \pi R C f_{0}} \arctan \left(2 \pi R C f_{0}\right)\right] \\
& \text { If }\left(2 \pi R C f_{0}\right)<<1, \text { this equation simplifies to: }
\end{aligned}
$$

$$
f_{\varphi=0} \approx-f_{0}\left(2 \pi R C f_{0}\right)^{2}=-f_{0}\left(\frac{f_{0}}{f_{-3 d B}}\right)^{2}
$$

These equations show that the amount of $\mathrm{f}_{\varphi=0}$ increases for higher center frequency, leading to more delay variation according to equation 5, which fits to the expectation.

Similarly, equation 13a is the result of $\mathrm{f}_{\varphi=0}$ for an LC delay segment and for $\left(2 \pi \sqrt{L C} f_{0}\right)<<1$, it reduces to equation $13 \mathrm{~b}$.

$$
\begin{gathered}
f_{\varphi=0}=f_{0}\left[1-\frac{1+\left(2 \pi \sqrt{L C} f_{0}\right)^{2}}{2 \pi \sqrt{L C} f_{0}} \cdot \arctan \left(2 \pi \sqrt{L C} f_{0}\right)\right] \\
f_{\varphi=0}=-f_{0}\left(2 \pi \sqrt{L C} f_{0}\right)^{2}
\end{gathered}
$$

Again, we see an increase of the $\mathrm{f}_{\varphi=0}$ for higher center frequency. 


\section{V. $\mathrm{F}_{\varphi=0}$ OF THE CASCADED DELAY BLOCKS}

Fig 7 shows the phase vs. frequency of two different delay blocks for the range between $\mathrm{f}_{0}$ and $\mathrm{f}_{0}+\Delta \mathrm{f}$. They have different group delay and $\mathrm{f}_{\varphi=0}$. Cascading them with buffers in between which omits the loading effect between them, or cascading them without buffer but with considering the loading effect of the next stage on the previous one results in a new delay block. We will now derive the $\mathrm{f}_{\varphi=0}$ for the cascade.

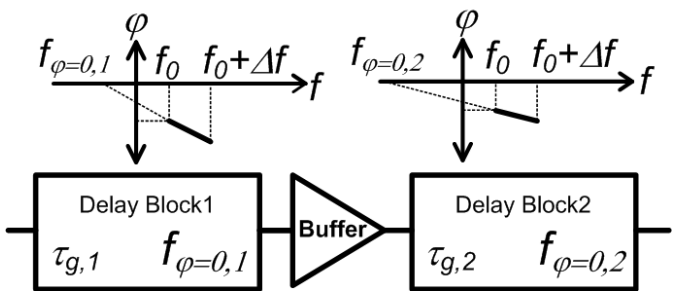

Figure 7. Cascading delay blocks

The group delay of the cascade is equal to the sum of the group delays of each individual block and its $f_{\varphi=0}$ will be found by adding two transfer functions of fig 7 and finding the frequency for which the extrapolated phase is zero. The result of $\mathrm{f}_{\varphi=0}$ for cascaded circuits is written in equation 14 .

$$
f_{\varphi=0, \text { cascade }}=f_{\varphi=0,1} \cdot \frac{\tau_{g, 1}}{\tau_{g, 1}+\tau_{g, 2}}+f_{\varphi=0,2} \cdot \frac{\tau_{g, 2}}{\tau_{g, 1}+\tau_{g, 2}}
$$

Equation 14 shows that if two delay blocks are the same, then $f_{\varphi=0}$ will be the same as for the individual blocks. Also, for the LC delay line in Fig 6a, in which the loading effect is already taken into account, the $\mathrm{f}_{\varphi=0}$ of the whole delay line is equal to that of each LC segment.

\section{SiMULATION RESUlTS:}

To examine the ability of the proposed criterion $f_{\varphi=0}$ to predict time delay variations over a frequency band, we continue with two examples of delay circuits: 1) an RC delay block and 2) an LC delay line. Suppose that both of them are designed for $\mathrm{f}_{0}=1 \mathrm{GHz}$ and we want to evaluate their time delay variation at $\pm 100 \mathrm{MHz}$ around $\mathrm{f}_{0}$. For both of the examples we use linear approximation of the phase vs. frequency transfer function and we will show that it results in a reasonable approximation of the circuit behavior.

Example 1: An RC time delay block (Fig 6a), with $\mathrm{R}=1 \mathrm{~K} \Omega, \mathrm{C}=490 \mathrm{fF}$, at $\mathrm{f}_{0}=1 \mathrm{GHz}$ has a time delay equal to $\tau_{\mathrm{d}}=200$ psec. The question is now; what are the time delays at $\mathrm{f}_{0} \pm(100 \mathrm{MHz})$ ?. Substituting these values in equation $11 \mathrm{a}$ and solving $\mathrm{f}_{\varphi=0}$ at $\mathrm{f}_{0}=1 \mathrm{GHz}$, we find $\mathrm{f}_{\varphi=0}=-3.28 \mathrm{GHz}$. The maximum time delay variation inside the frequency band is found from equation 5. In Fig 8, curve (a) shows $\varphi$ vs. f for the simulation results of the RC delay block. Table la shows the delays which results from calculation, simulation and the relative error of calculation results to simulation results.

Example 2: An LC delay line (Fig 6 b), with $\mathrm{L}=1 \mathrm{nH}$, $\mathrm{C}=253 \mathrm{fF}$, at $\mathrm{f}_{0}=1 \mathrm{GHz}$ after the $12^{\text {th }}$ cell shows a delay equal to $\tau_{\mathrm{d}}=204.2 \mathrm{psec}$ (a delay value near to example 1). Again we want to find the time delays at $\mathrm{f}_{0} \pm(100 \mathrm{MHz})$. Equation $13 \mathrm{~b}$ can be used as $2 \pi(\mathrm{LC}){ }^{0.5} \mathrm{f}_{0}=0.1<<1$. We find the value of $\mathrm{f}_{\varphi=0}$ for every $\mathrm{LC}$ delay cell which is loaded with $\mathrm{Z}_{0}=(\mathrm{L} / \mathrm{C})^{0.5}$. For every LC cell the calculation result is $\mathrm{f}_{\varphi=0}=-10 \mathrm{MHz}$. Because the loaded LC cells are cascaded then equation 14 can be used for finding the overal $\mathrm{f}_{\varphi=0}$ of the LC delay line which results in: $\mathrm{f}_{\varphi=0 \text {, Circuit }}=\mathrm{f}_{\varphi=0 \text {,every cell }}=-10 \mathrm{MHz}$. In Fig 8 , curve (b) shows $\varphi$ vs. $\mathrm{f}$ for the simulation results of the LC delay circuit. Table $1 \mathrm{~b}$ shows the delays which results from calculation, simulation and the relative error between them.

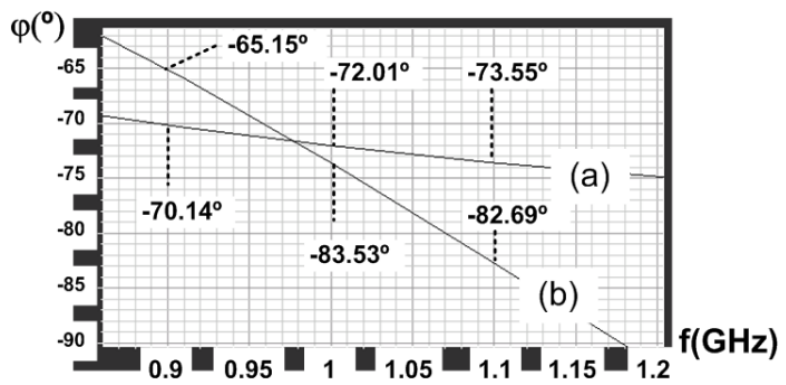

Figure 8. Curves (a) and (b) shows $\varphi$ vs. $f$ transfer function of an RC delay cell and an LC delay circuit respectively

\begin{tabular}{|c|c|c|c|}
\hline RC delay cell & $0.9 \mathrm{GHz}$ & $1 \mathrm{GHz}$ & $1.1 \mathrm{GHz}$ \\
\hline $\begin{array}{c}\tau_{\mathrm{d}} \text { (psec) } \\
\text { Calculation }\end{array}$ & 215.4 & 200 & 184.6 \\
\hline $\begin{array}{c}\tau_{\mathrm{d}} \text { (psec) } \\
\text { Simulation }\end{array}$ & 216.4 & 200 & 185.7 \\
\hline Error $(\%)$ & $0.5 \%$ & $0 \%$ & $0.6 \%$ \\
\hline
\end{tabular}

(a)

\begin{tabular}{|c|c|c|c|}
\hline LC delay circuit & $0.9 \mathrm{GHz}$ & $1 \mathrm{GHz}$ & $1.1 \mathrm{GHz}$ \\
\hline $\begin{array}{c}\tau_{\mathrm{d}} \text { (psec) } \\
\text { Calculation }\end{array}$ & 204.4 & 204.2 & 204 \\
\hline $\begin{array}{c}\tau_{\mathrm{d}}(\mathrm{psec}) \\
\text { Simulation }\end{array}$ & 201.1 & 204.2 & 208.8 \\
\hline Error(\%) & $-1.6 \%$ & $0 \%$ & $2.3 \%$ \\
\hline
\end{tabular}

Table 1. comparison of the calculation and simulation results for (a) $\mathrm{RC}$ delay cell (b) LC delay circuit

The results show that the formula predicts the simulation with quite acceptable accuracy (a few \% over $+/-10 \%$ frequency variation around the center frequency).

\section{CONCLUSION}

This paper shows that group delay alone is not sufficient for characterizing the delay variation in delay blocks and proposes $\mathrm{f}_{\varphi=0}$ as an additional criterion. The combination of $\mathrm{f}_{\varphi=0}$ with the group delay allows for estimating the delay variations over a certain frequency band around center frequency $\mathrm{f}_{0}$. Equations for $\mathrm{f}_{\varphi=0}$ for an RC delay cell and $\mathrm{LC}$ delay section have been derived and compared to simulations, showing the prediction is within few percents over $10 \%$ frequency variation.

\section{REFERENCES}

[1] Robert J. Mailloux., "Phased Array Antenna Handbook, Second Edition" (Artech House Antennas and Propagation Library,2005)

[2] Kendall L.Su., "Analog filters" (Chapman \& Hall press, 1996)

[3] K. D. D., "Are group delay time and/or phase delay time useful parameters for defining low distortion transmission." Communication, IEEE Transaction on., vol. COM-21. pp. 14461448, Dec. 1973

[4] Mueller, M., "Signal Delay" Communications, IEEE Transactions on Volume 23, Issue 11, Page(s):1375 - 1378 , Nov 1975

[5] Frank Ellinger.,"Radio Frequency Intergrated Cicruits and Technologies" (Springer 2007) 